\title{
Improving Inter-Professional Communication among Medical and Dental Providers Treating Bisphosphonate Therapy Patients May Reduce the Risk of Osteonecrosis of the Jaw
}

\author{
Tena Dischler ${ }^{1, *}$, Jeffrey L. Alexander², Denice Curtis ${ }^{2}$, Michael Markson³ \\ ${ }^{1}$ Private General Dental Practice, 10814 N $71^{\text {st }}$ Place, Scottsdale, Arizona 85254 \\ ${ }^{2}$ Department of Interdisciplinary Health Sciences, A.T. Still University, 5850 East Still Circle, Mesa, Arizona 85206 \\ ${ }^{3}$ Private Endodontic Practice, 10802 North $71^{\text {st }}$ Place, Scottsdale, Arizona 85254 \\ *Corresponding author: tdischler@atsu.edu
}

Copyright (C) 2014 Horizon Research Publishing All rights reserved.

\begin{abstract}
While large clinical trials have shown rare adverse side effects in post-menopausal women taking uninterrupted oral bisphosphonates (BPs), there is mounting evidence that longer-term treatment may be associated with osteonecrosis of the jaw (ONJ). There is a paucity of information regarding the causal relationship between extended oral BP treatment and ONJ, and the safety of non-surgical therapy of apical lesions and length of treatment in women on long-term BPs. We describe a post-menopausal woman on long-term oral BPs who developed ONJ without any contributory risk factors. Quantitative data was gathered through review of the patient's medical record. Qualitative data was gathered from face-to-face interviews with patient and clinician. Analysis of the data revealed the patient did not feel it was important to report her use of BPs. Given the wide usage of antiresorptives dental clinicians should manage all post-menopausal women with the assumption they currently or have recently been treated with BPs. There is a need for a collaborative effort by medical and dental practitioners to reduce risk of ONJ, ensure proper BP dosing and length of treatment, identify contributory risk factors, and explore alternate modalities, such as $\mathrm{Ca}(\mathrm{OH})_{2}$ multi-visit palliative pulpectomy, to avert $\mathrm{ONJ}$ and improve treatment outcomes.
\end{abstract}

Keywords Bisphosphonates. Antiresorptives. Osteonecrosis of the Jaw. ONJ. Pulpectomy

\section{Introduction}

Approximately 2500 cases of osteonecrosis of the jaw (ONJ) have been reported in the literature since 2003 [1]. Each year, 30 million prescriptions for oral bisphosphonates are written in the U.S. alone [2]. No causal relationship has been established between chronic, long-term oral BP therapy and ONJ, but there is an urgent need to close the knowledge gap to allow clinicians to identify the condition, determine the cause, prevent bone necrosis, and identify patients at risk. The human cost to patients with ONJ is immeasurable due to severe pain, loss of function, disfigurement, and a long, costly recovery time.

Bisphosphonates have been used to treat skeletal fragility effectively in post-menopausal women for over a decade. Studies reported by Gladnick [3] and Odvina et al. [4] suggest long-term uninterrupted BP therapy suppresses bone turnover, increasing crystallinity, and impedes repair of micro-damaged bone. The Federal Drug Administration (FDA) in October 2010 added a warning label regarding possible risks of atypical femur fracture with the use of Fosamax (Merck) and Actonel (Procter \& Gamble Pharmaceuticals). The revised label states the optimal duration of use has not been determined [5]. A task force set up by the American Society of Bone and Mineral Research in September 2010 found $94 \%$ of 310 atypical femur fractures were associated with oral bisphosphonate therapy $>5$ years $[5,6]$.

Concern is growing in medical and dental communities that there appears to be a parallel between atypical femur fracture and ONJ. Suppressing osteoclastic activity over long periods produces dense, homogeneous bone, prone to injury with minimal assault. Although not all dental patients develop ONJ after invasive surgical procedures, evidence suggests incidence of ONJ may be higher due to a lack of recognition by dental clinicians and underreporting [7]. Studies have suggested that onset of ONJ occurs from micro-damage to the alveolar bone during mastication, or bone defects resulting from extraction that cannot be effectively repaired [8]. Another possible explanation is the 
decrease in the vascularization of the bone, seen in long-term therapy with oral BPs and the body's inability to repair the injured sites [3,9]. Endodontic therapy has been the preferred treatment for dental apical infection, as opposed to surgical extraction in BP treated populations. As the numbers of patients treated with BPs, and length of treatment increases, higher numbers of $\mathrm{BP}$ related $\mathrm{ONJ}$ is being reported in endodontically treated patients [10]. Therefore, there is a need to improve medical history questionnaires and explore new modalities to decrease the threat of ONJ in this cohort.

\subsection{Objective}

The purpose of this case report was to describe the unique clinical events that unfolded in everyday clinical practice regarding a post-menopausal woman on long-term oral BPs that developed ONJ without any known significant risk factors. This case builds an argument for the need to reevaluate current protocols and proposes procedures to advance patient safety, mitigating the risk of ONJ in this cohort.

\section{Materials and Methods}

The research conducted was a retrospective, descriptive case report. An analytical review of the medical record of one patient diagnosed with ONJ in October 2009 was conducted in the primary investigator's dental practice. The case was a healthy, post-menopausal woman with an unremarkable health history, without any known contributory risk factors, other than long-term oral BP use. The participant was alert in three dimensions. She admitted no use of alcohol or tobacco. The participant was Caucasian American, college-educated, middle class, from a moderately populated area in the southwest United States.

Chart reviews were conducted for 60 post-menopausal women seen in the dental practice in 2013 to assess whether or not they reported use of bisphosphonates on their medical questionnaire.

\subsection{Qualitative Assessment}

Incidents and events from the patient's medical record were recorded. After retrieving the data and conducting a preliminary analysis of the patient's medical history, a face-to-face, semi-structured interview was conducted. The primary investigator was compelled to understand why the participant did not disclose to the dental clinician that she was undergoing long-term oral bisphosphonate therapy. Three open-ended questions were posed to the participant during the interview:

1. Who prescribed the oral bisphosphonate therapy and for what reason?

2. Did the prescribing physician review the mechanism of action of bisphosphonates and any possible side effects?
3. Why did the participant not disclose taking oral bisphosphonates to the dental clinician during the past eight years?

Preliminary analysis of the data with the participant prompted a similar interview with the patient's dentist to explore what could have been done to prevent the onset of ONJ.

1. What improvements can be made to patient reporting of BP therapy in everyday clinical practice?

2. What can be done to improve the clinician's knowledge regarding ONJ to facilitate earlier diagnosis?

3. What could reduce the risk of ONJ in post-menopausal women on antiresorptive therapy?

\subsection{Measurement Tools}

Outcome measurement of the apical lesion for patients undergoing non-surgical root canal therapy was categorized into healed, healing, or not healed, pursuant to the current guidelines of the American Association of Endodontists [12]. Clinical evaluation, symptoms, pulpal testing (Vitapulp, Pelton \& Crane), appearance, and radiological evaluation (Gendex 765DC, Gendex Dental, Orthopantomograph OP200D, Instrumentarium, Dental) were other instruments used for measurement (Appendix A). Pain was assessed on an ordinal scale of $0-10$, with 10 being the severest pain [13]. Precision pain measurement was not required for meaningful interpretation because the presence of pain alone would be a determinant for treatment.

\subsection{Qualitative Data Analysis}

Qualitative data analysis included (a) reading through the participant's medical record and transferring the information to a custom data collection form to gain a broad picture of the data, (b) examining the data for information related to the study purpose, (c) coding and labeling the units of information to capture the meaning, (d) interpreting emerging themes related to the study purpose, (e) verifying the trustworthiness of the findings (triangulation), and (f) describing the findings in both a textual format and visual displays [14].

Face-to-face interview notes were reviewed with the participant by the investigator and the observer for accuracy. Face-to-face interview notes were identified conceptually, labeled, and coded. Coding involved integrating the categories into a larger theoretic scheme and organizing them around an explanatory concept. The analysis was inductive and attempted to explain the phenomena studied. Methodological triangulation was used for qualitative analysis to assure trustworthiness. The conceptual framework interpretation and analysis were compared to the literature to support the study theory. Notes from the face-to-face interview conducted with the participant's clinician were evaluated using the same process as with the notes from the participant interview. 


\subsection{Quantitative Data Analysis}

Quantitative analysis assessed the number of patients in this cohort previously not reporting using BP therapy on their medical history questionnaire.

\section{Results}

Thirty-one post-menopausal women of 60 reviewed, did not report use of bisphosphonates on their medical questionnaire. Upon returning for preventive appointments, when questioned specifically regarding bone repair medications, admitted receiving anti-resorptive therapy. Of the charts reviewed, $52 \%$ of post-menopausal women did not disclose the use of BP therapy suggesting a significant number of patients are not aware of the importance of reporting oral BP therapy to the treating dentist.

The participant who developed ONJ was an 83-year-old healthy post-menopausal woman with an unremarkable health history without any known contributory risk factors for ONJ except long-term use of oral BPs. The participant reported regular physical activity, expressed a high quality of life, continues to travel, enjoys reading, frequent social gatherings, and actively involved in her church. The patient entered the dental practice in 1988 and had maintained a high level of oral hygiene and followed up with timely preventive visits.

\subsection{Development of Osteonecrosis of the Jaw}

The patient developed a periapical radiolucency in tooth \#28 in October 2009. A radiological survey was performed and revealed a possible abscess and external root resorption (Figure 1). A pulp vitality test indicated the nerve did not respond to normal stimulus, was compromised, and required endodontic therapy. Tooth \#29 was also tested and found to be vital within normal limits. Endodontic treatment was performed on Tooth \#28, external root resorption repaired, and the prognosis favorable. Pulpal therapy was completed in one visit using gutta percha and AHP sealer, which is the standard of care for obturation materials [12] (Figure 2).

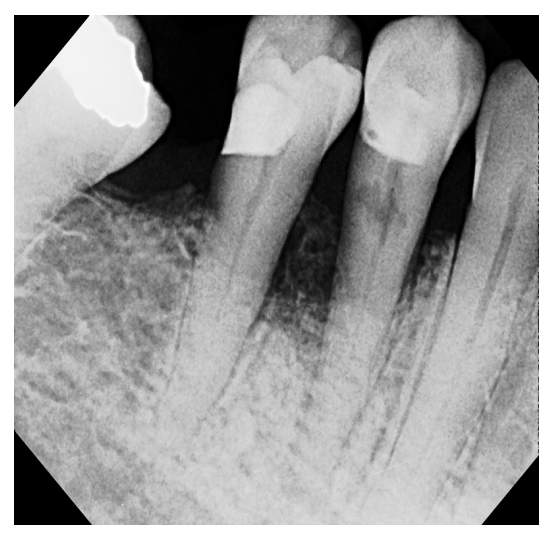

Figure 1. Initial pre-op \#28 10-13-09

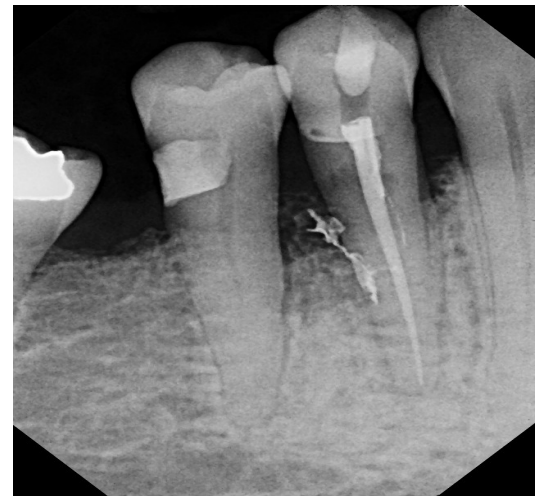

Figure 2. Post-op \#28 Single visit endo 10-20-09

Five months post-endodontic therapy, the patient developed mobility in teeth $\# 28$ and \#29. The clinician determined the probable cause of the mobility was excessive pressure and trauma from clenching or mastication. The occlusion was adjusted to relieve excessive forces and the teeth splinted together to provide stability to the area since the patient masticated primarily on the right side. Ten months following endodontic therapy, the patient presented with increased mobility and purulent suppuration in teeth $\# 28$ and \#29 (Figures 3).

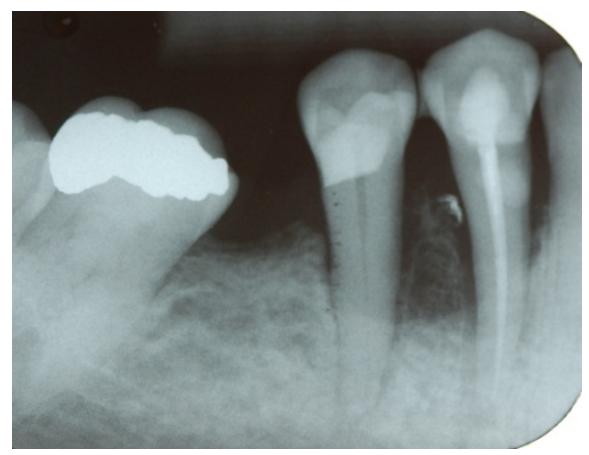

Figure 3. Bone loss and mobility 2-8-10

Radiological evaluation confirmed the periapical radiolucency had increased to 1.5 centimeters. After an extensive patient-clinician consultation, it was determined both teeth would be extracted as there was no further treatment options. Patient's vitals remained within normal limits and no contributory health factors were identified. The clinician performed routine, simple extractions and dismissed the patient with standard post-operative instructions. During this time, the patient described pain levels no greater than four, according to the visual analog scale of $0-10$. Six months post extraction, the patient presented with an area of exposed, necrotic bone at the surgical site (Figure 4).

Suspecting a diagnosis of ONJ, the clinician questioned the patient specifically regarding the use of BPs, such as Fosamax or Boniva. The patient admitted that she had forgotten to disclose she had been taking Fosamax for approximately eight years. Upon learning of the long-term bisphosphonate use by the patient, supported by a 
radiological survey, and the exposed bone sequestrum $>8$ weeks, confirmed a diagnosis of ONJ. The necrotic bone sequestrum was removed and the patient was placed on antibiotics and Chlorhexidine mouth rinse. The patient consulted with her primary care physician $(\mathrm{PCP})$ regarding the development of ONJ and after evaluation, a mutual decision was made by the patient and the PCP to discontinue oral BP therapy. Three months post termination of BP therapy, the migration of the lesion ceased and early signs of healing became evident on radiographs. The patient was followed-up for 36 months from the onset of the disease to a fully healed condition and reported overall general wellness. (Figure 5).

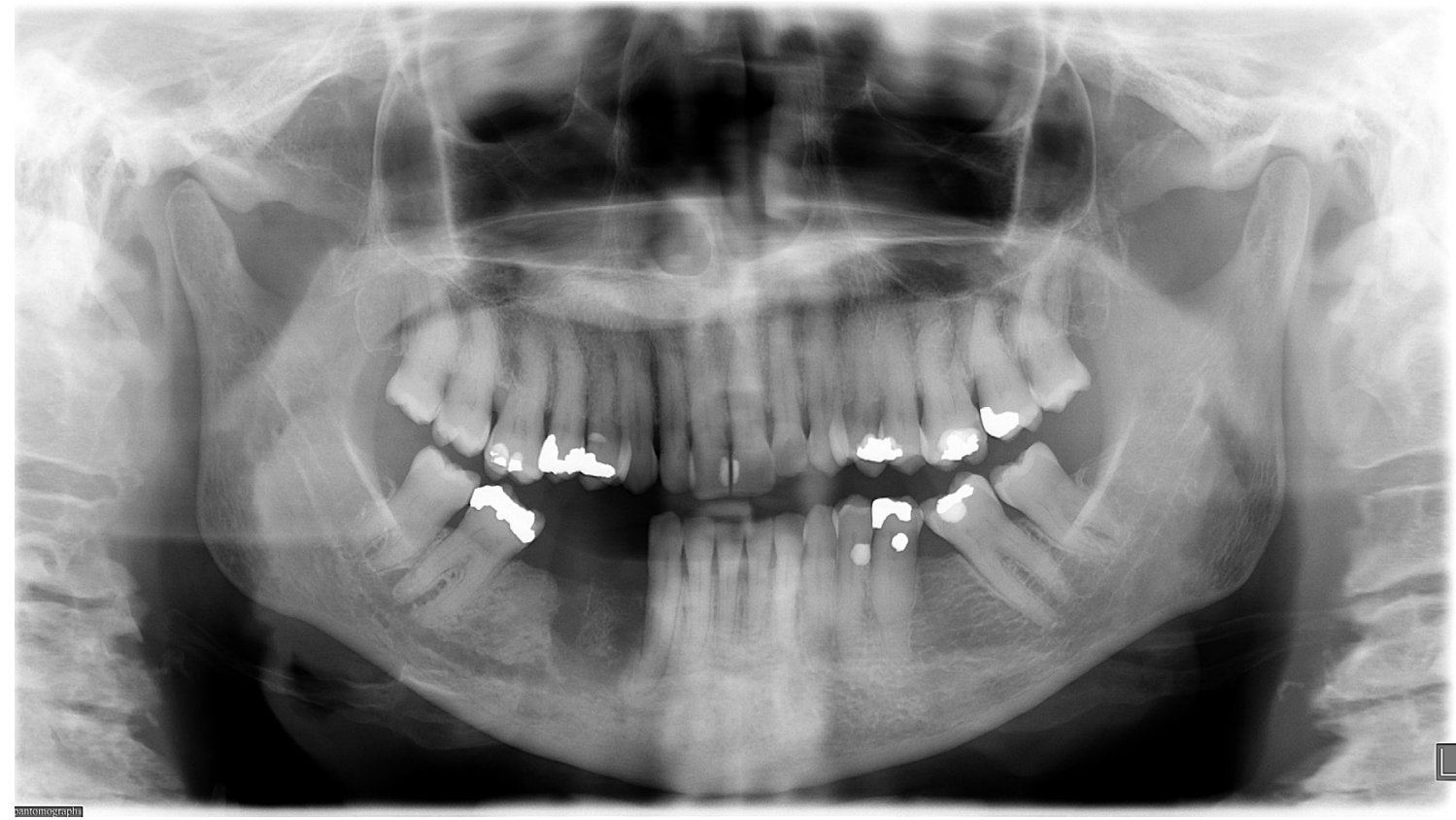

Figure 4. Post-surgical x-ray 3-22-11, initial diagnosis of ONJ (Not Healed)

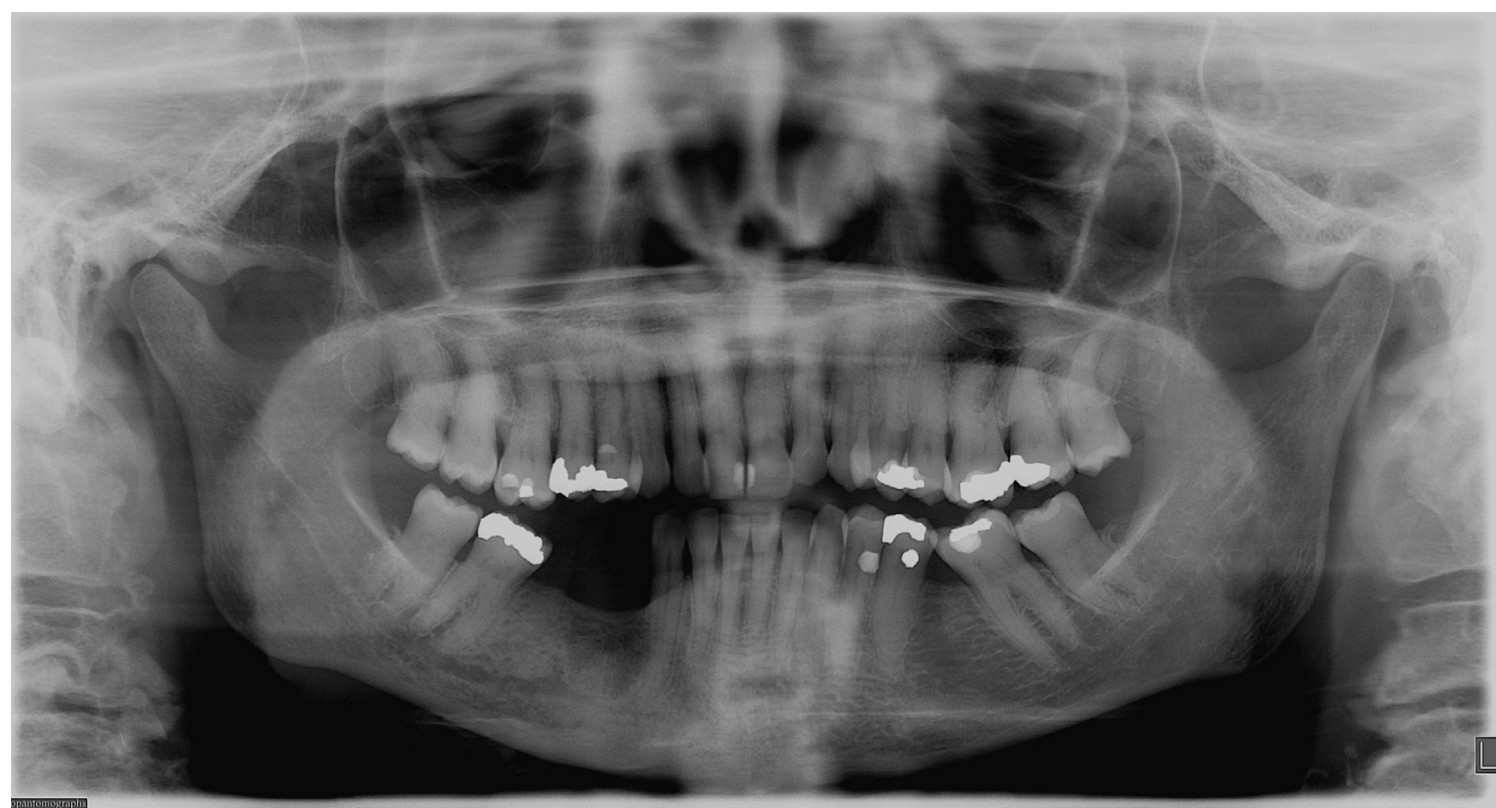

Figure 5. Post-surgical x-ray 9-11-12, ONJ (Healed) 


\subsection{Emerging Themes}

The following emerging themes were revealed: the lack of the PCP educating the patient about the use of BPs and possible side effects. The physician never discussed the need for the patient to inform her dentist of her treatment with BPs. The participant stated, "I did not realize the importance of disclosure of Fosamax prior to dental procedures." And "I thought Fosamax worked like calcium, only better, to strengthen my bones." Additionally, she stated, "the physician did not consistently test my bone density"

A face-to-face interview with the treating dentist revealed the need for a clear definition of ONJ and improved education of the dental community. The clinician is quoted, "because of the long half-life of these antiresorptive drugs, there is a need to assume all post-menopausal women have a history of BP use prior to surgical interventions." In addition, "dentists need to explore new modalities to reduce the risk of ONJ in this cohort." The clinician also stated there is "the need to improve patient medical questionnaires asking for current or past use of bisphosphonates, clearly identifying common types of BPs like Fosamax or Boniva." Lastly, the dentist indicated, "improved communication between practitioners for optimal treatment for patients undergoing BP therapy."

\subsection{Calcium Hydroxide Palliative Pulpectomy}

If the clinician had knowledge of the patient's long-term bisphosphonate use and a clearer etiology of the disease, a timely diagnosis may have precipitated earlier preventive modalities that might have reduced the risk of ONJ, such as multi-visit palliative pulpectomy using Calcium Hydroxide $\mathrm{Ca}(\mathrm{OH}) 2[15,25,26,34]$.

\section{Discussion}

Studies by Gladnick et al. [3], and Burr and Allen [16] have shown BPs are extremely effective in managing skeletal fragility by suppressing osteoclastic activity and osseous turnover. After uninterrupted extended use, without proper screening for bone mineral density, there appears to be an increasing accumulation of over compacted, avascular crystalline bone in both femurs and the oral cavity. These osseous tissues can be damaged by minimal trauma and produce skeletal susceptibilities that these drugs were originally formulated to mitigate $[3,16]$. The findings of this case report support the theory that oral BP therapy, prescribed for a long uninterrupted period, may result in an increased risk of ONJ. Diagnosed late, ONJ can cause facial disfigurement, require years of costly palliative treatment, and, in some cases, mortality [17].

Currently, few studies in the literature adequately address the incidence of ONJ associated with the use of BPs. Weaknesses of these studies are they do not account for the differences in dosages of BP, and lack assessment of BP patients greater than three years [18]. There is a paucity of information to identify ONJ, reluctance of medical professionals in non-institutional settings to report necrotic bone disease, and a lack of utilization of the entire health care team in developing optimal treatment plans for BP patients. Many dental clinicians remain uninformed concerning the upsurge in documented side effects of these antiresorptive compounds [19]. This results in patients receiving little or no education from their PCPs, remaining ignorant regarding complications when invasive bone procedures are undertaken. Patients do not realize the importance of sharing this information with the practitioners that are providing their healthcare. Time restrictions during clinical visits may impede extensive questioning of patients regarding the numerous brands of antiresorptives. The investigators suggest a more specific medical questionnaire in combination with the clinical interview to increase successful treatment outcome.

Almazrooa and Woo [20] defined BP induced ONJ as the exposure of dead bone in the oral maxillofacial region in patients taking BPs, which persists for more than eight weeks. In a large institutional study by Sedghizadeh et al. [7], 4\% ( $n$ $=9$ ) of the 208 patients treated at the University of Southern California dental clinic using alendronate had active ONJ, suggesting BP-associated necrotic jaw is more common than previously thought.

Since not all individuals on BP therapy develop ONJ, other risk factors include trauma from mastication, chemicals such as cancer-preventive agents, glucocorticosteroids, poor oral health, or dental surgery [21]. Crystalline or brittle bone has been associated with micro-fractures and increased skeletal fragility in dogs. Burr and Allen [16] found that after three years of uninterrupted oral BP treatment, bone matrix necrosis occurred in 33\% of the dogs receiving $1.0 \mathrm{mg} / \mathrm{kg} /$ day of alendronate, equal to the therapeutic dosage in osteoporotic women, but paltry human research does not support these higher percentages. While the results of this study implicate uninterrupted BP therapy and the development of $\mathrm{ONJ}$, these findings require extrapolation of data from animals to humans. Conversely, the optimum duration of BP treatment has not been established, or the association of adverse events in post-menopausal women with osteoporosis [23].

In a retrospective clinical study, Hsiao et al. [12] examined 34 cases of healing periapical lesions, in patients taking chronic oral BPs after root canal therapy, compared to a control group of patients not taking oral BPs. Radiographic and clinical evaluation indicated $26.5 \%$ of patients taking BPs had non-healing periapical lesions after 10 months, compared to $18.4 \%$ in the control group. Hsiao et al. concluded that endodontic therapy was safer than surgical extraction in treating patients on extended BPs. Evidence is surfacing those patients on long-term, oral BP therapy presenting with spontaneous apical radiolucency might still be at significant risk of ONJ when treated with endodontic therapy [10].

This case report is remarkable in that the original radiolucent periapical lesion occurred without trauma or 
increased risk factors, with the exception of extended BP therapy, diagnosed as a simple pulpal abscess, and treated according to American Dental Association (ADA) guidelines [24]. The placement of $\mathrm{Ca}(\mathrm{OH})_{2}$ helps heal micro-fractures, decreases inflammation, and has bactericidal properties, further reducing the risks of necrotic bone formation [25]. In a longitudinal study of 78 non-vital teeth treated with $\mathrm{Ca}(\mathrm{OH})_{2}$, Vernieks and Messer, [26] concluded it accelerated the natural healing function of the periapical tissues. Carrotte [15] reported $\mathrm{Ca}(\mathrm{OH})_{2}$ was effective in repairing root fractures, open apices, perforations, and root resorption.

The authors of this case report suggest it would be appropriate to stabilize this cohort with multi-visit $\mathrm{Ca}(\mathrm{OH})_{2}$ palliative pulpectomy, and continue follow-up for approximately 8 to 12 weeks, evaluating for radiographic evidence of apical healing. In severe apical infections that are slow healing, dentists should consider replacing the $\mathrm{Ca}(\mathrm{OH})_{2}$ every 8 to 12 weeks until healed. Further research is needed however, to establish the efficacy of this treatment practice.

\subsection{Implications}

Cases of BP-related ONJ are underreported likely due to the lack of knowledge of symptoms and mechanisms of action of BPs and problematic treatment of ONJ. There is also a reluctance of dental practitioners to explore different modalities to diminish the risks of the disease [18]. Because PCPs are the first contact in treating this cohort for skeletal fragility, it is important for them to educate patients regarding the use of long-term oral bisphosphonates and possible side effects. There is also a need in the medical community to determine the optimal length of BP therapy in this cohort, and communicate with dental practitioners to improve treatment management. Dental clinicians should treat all post-menopausal women with the assumption they currently or have recently undergone antiresorptive therapy and still have a significant reservoir of BPs in the skeletal system. Palliative pulpectomy with $\mathrm{Ca}(\mathrm{OH})_{2}$, with concurrent antibiotic therapy, should be the initial treatment in this cohort, when confronted with infected tooth apices. Multi-visit therapy with $\mathrm{Ca}(\mathrm{OH})_{2}$ would be appropriate to decrease risk of ONJ due to its bactericidal effects and accelerated bone healing properties in periapical tissues $[15,25,26,34]$.

We believe it necessary for dental clinicians to be better educated regarding ONJ's manifestations and to question patients specifically regarding BP use. It is becoming more apparent, as seen in the primary investigator's dental practice, more than half of post-menopausal women with a history of bisphosphonate therapy, are not reporting its use on the written medical questionnaire. Patients must understand how this class of drugs affects any invasive hard tissue surgical procedures. Dental clinicians, when confronted with external root resorption of unknown etiology in this cohort, should suspect the possibility of the patient developing ONJ if invasive dental procedures are contemplated. Consequently, there is a need to explore new modalities to reduce the risk of ONJ in post-menopausal women.

According to the literature [7], surgical extraction of teeth in chronic BP treated patients with apical radiolucency results in a significantly higher risk of developing ONJ as compared to endodontic treatment. However, this case is an example that traditional endodontics may not completely avert the onset of ONJ, and removing affected pulp tissue, performing sedative palliative pulpectomy, waiting for tissue healing and inflammation reduction could improve outcomes and diminish the risk of bone necrosis.

According to Vogt-Ferrier, Hugentobler, Uebelhart, Tramèr, and Rollason [27], stopping BP therapy in patients with osteoporosis does not seem to aggravate the condition and may be beneficial. BP accumulates in the bones and remains at therapeutic levels for years after treatment is terminated [28]. Therefore, it is reasonable to ask at what point treatment should terminate. Watts and Diab [28] posit BPs create a bone reservoir leading to continued release of therapeutic dosages, suspected to last up to five years, and an anti-fracture efficacy for two years or more. Watts and Diab suggest patients at mild risk of fracture could stop treatment after five years, and discontinue treatment as long as the bone mineral density (BMD) value remains stable. Screening programs for bone fragility must depend on best available evidence, rather than pharmaceutical industry promotion, or unsupported empirical observations, in view of the recent controversy in the medical community for over testing and over treating [29].

The primary outcomes reported by Gourlay et al. [30] were that osteoporosis was diagnosed in less than $10 \%$ of women with normal test scores at baseline and $5 \%$ of women with a diagnosis of mild osteopenia at first assessment over a 15 -year period. This finding suggested during this time interval, there was a very low risk of this cohort transitioning to osteoporosis. The authors' findings were consistent with studies by Cauley et al. [31].

\subsection{Palliative Pulpectomy}

Numerous studies have suggested that prolonged use of oral bisphosphonates reduces the healing properties of bone due to the long half-life of these antiresorptive drugs that remain at therapeutic levels in the skeletal system for up to five years [30]. There is additional evidence that shows suppression of skeletal healing with continued bisphosphonate use [32,33]. Although the protocol for $\mathrm{Ca}(\mathrm{OH})_{2}$ pulpectomy is when the nerve is determined to be necrotic, dentists should anticipate an increased level of bone damage due to micro-fractures, in post-menopausal women on extended oral BPs, and multi-visit palliative pulpectomy should be the first line of defense to reduce the risk of ONJ. There is a concern among investigators that masticatory forces may precipitate $\mathrm{ONJ}$ in patients on long-term oral BPs creating micro-fractures in avascular, crystalline bone, damaging the supporting alveolar structures [16]. Severity of 
injury to dental tissues may be exhibited in the pulpal tissue as internal or external resorption and pulp necrosis.

Carrotte, [15], Soares, et al. [25], Vernieks and Messer [26], and Aguiar, Andrade-Mendes, \& Camara, [34] conclude the action of $\mathrm{Ca}(\mathrm{OH})_{2}$ accelerated the natural healing function of the periapical tissues. These findings demonstrate the efficacy of $\mathrm{Ca}(\mathrm{OH})_{2}$ palliative pulpectomy and would very likely be an appropriate treatment modality to consider in reducing the risk of ONJ in this cohort, and deserves further research.

\subsection{Limitations}

The investigators understand this is simply a description of a clinical phenomenon and the related experiences of one individual affected with ONJ, so generalizability may not be possible, and up to the individual reader. However, the time may be appropriate not to minimize the debilitating side effects of prolonged use of oral BPs and consider out-of-the-sphere therapies to curtail and possibly avoid serious pathological consequences. The investigators' hope is that by relating this clinical case, supported by the literature, medical and dental professionals and the scientific community will transfer these findings to their own settings. Further research is necessary to determine optimal duration of treatment of BPs and explore other modalities to reduce the risk of oral BPs' adverse effects.

\section{Conclusion}

Patients and practitioners are demanding information as awareness increases of an association between oral BP use and ONJ. There appears to be a parallel mechanism between over-compressed crystalline bone in atraumatic femur fractures and ONJ. Sedghizadeh et al. [7] have shown the incidence of ONJ appears to be significantly underreported. Some explanations include the lack of the PCP educating patients as to the mechanism of bisphosphonates, risk factors, the need to report BP therapy to the dental practitioner, and reluctance by practitioners to report cases of ONJ to the Center for Disease Control and Prevention.

There is a need for a collaborative effort by the medical and dental team to improve inter-professional communication, ensure proper BP dosing, evaluate length of treatment, identify contributory risk factors, vigilance for early diagnosis, and exploring enhanced modalities to avert bone necrosis. The dental practitioner is in an advantageous position to educate patients regarding risks of oral BPs and their association with surgical dental procedures. The dental community needs to improve policies and procedures for identifying common antiresorptive drugs, understand signs and symptoms of ONJ, and evaluate other clinical procedures for treatment of affected dentition in this cohort. This study proposes multi-visit palliative pulpectomy be included in future investigations to reduce the risk of ONJ in post-menopausal women requiring endodontic therapy. The investigators hope the findings reported from this case will stimulate further research in the recognition, treatment, and prevention of this perplexing pathology.

\section{Conflict of Interest}

All authors are free of conflicts of interest, relationships to products or companies mentioned, or related to the subject matter of the article. Funding was not required for this study.

\section{REFERENCES}

[1] R. Wynn 2408 patient cases of bisphosphonate-associated osteonecrosis of the jawbone: The most extensive review to date. Lexicomp Online for Dentistry. 2010. Retrieved from http://www.lexi.com/individuals/dentistry/newsletters.jsp?id $=$ september_11

[2] N. Masoodi Oral bisphosphonates and the risk for osteonecrosis of the jaw. Br J Med Pract. 2009;2(2):11-15. Retrieved from http://www.bjmp.org/content/oral-bisphosph onates-and-risk-osteonecrosis-jaw

[3] B. Gladnick, E. Donnelly, D. Lorich, A. Unnanuntana, E, DiCarlo, S. Doty, A. Boskey, J. Lane. The effects of long-term bisphosphonate use on bone quality. AAOS, Podium Presentations. 2010;(Podium No. 339). Retrieved from

http://www3.aaos.org/education/anmeet/anmt2010/podium/p odium.cfm?Pevent=339

[4] C. Odvina, J. Zerwekh, D. Rao, N. Maalouf, F. Gottschalk, C. Pak. Severely suppressed bone turnover: A potential complication of alendronate therapy. J Clin Endocrinol Metab. 2005;90(3):1294-1301. Retrieved from http://jcem.endojournals.org/content/90/3/1294.long

[5] N. Masoodi. Bisphosphonates and atypical femur fractures. $\mathrm{Br}$ J Med Pract. 2010;3(1):311. Retrieved from http://www.bjmp.org/content/bisphosphonates-and-atypical-f emur-fractures

[6] D. Black, A. Schwartz, K. Ensrud, J. Cauley, S. Levis, S. Quandt, S. Satterfield, R.Wallace, D. Bauer, L. Palermo, L. Wehren, A. Lombardi, A. Santora, S. Cummings. Effects of continuing or stopping alendronate after 5 years of treatment. JAMA. 2006;296(24):2927-2938. doi:10.1001/jama.296.24. 2927

[7] P. Sedghizadeh, K. Stanley, M. Caligiuri, S. Hofkes, B. Lowry, C. Shuler. Oral bisphosphonate use and the prevalence of osteonecrosis of the jaw: An institutional inquiry. JADA. 2009;140(1):61-66. Retrieved from http://jada.ada.org/content/140/1/61.full

[8] R. Blanchaert, C. Harris. Bisphosphonate-related osteonecrosis of the jaw. Medscape. 2011. Retrieved from http://emedicine.medscape.com/article/1447355-overview

[9] J. Schilcher, K. Michaëlsson, P. Aspenberg. Bisphosphonate use and atypical fractures of the femoral shaft. N Engl J Med. 2011;364(18):1728-1737. doi:10.1056/NEJMoa1010650

[10] A. Sarathy, S. Bourgeois, G. Goodell. 
Bisphosphonate-associated osteonecrosis of the jaws and endodontic treatment: Two case reports. J Endodon. 2005;31(10):759-763.

doi:10.1097/01.don.0000182737.09980.2c

[11] S. LeFort. The statistical verses clinical significance debate. Image, McGill University School of Nursing. 1993;25(1):57-62.

[12] A. Hsiao, G. Glickman, J. He. A retrospective clinical and radiographic study on healing of periradicular lesions in patients taking oral bisphosphonates. J Endodon. 2009; 35(11):1525-1528. doi:10.1016/j.joen.2009.07.020

[13] L. Portney, M. Watkins. Principles of measurement. In: Portney L, and Watkins M (eds) Foundations of Clinical Research: Applications to Practice (3rd ed.), 2009. Pearson, Prentice-Hall, Upper Saddle River, NJ, pp 63-75.

[14] W. Pitney, J. Parker. Collecting and analyzing qualitative data. In Pitney W, and Parker J (eds) Qualitative Research in Physical Activity and the Health Professions, 2009. Human Kinetics, Champaign, Ill, pp 41-60.

[15] P. Carrotte. Endodontics: Part 9 Calcium hydroxide, root resorption, endo-perio lesions. $\mathrm{Br}$ Dent J. 2004;197(12):735-743. doi:10.1038/sj.bdj.4811897

[16] D. Burr, M. Allen. Mandibular necrosis in beagle dogs treated with bisphosphonates. Orthod Craniofac Res. 2009;12(3):221-228. doi:10.1111/j.1601-6343.2009.01456.x

[17] A. Pogrel. Oral pathology update presentation. Arizona Dental Association Western Regional Dental Conference Lecture, (2011, March).

[18] M. Jeffcoat. Safety of oral bisphosphonates: Controlled studies on alveolar bone. Int J Oral Maxillofac Implants. 2006;21(3):349-353. Retrieved from

http://www.implantsforteeth.com/13-1.pdf

[19] G. Goodell. BP-associated ONJ-Fall Newsletter 2012. AAE. 2012; Fall. Retrieved from http://www.aae.org/uploadedFiles/Publications_and_Researc $\mathrm{h} /$ Endodontics Colleagues for Excellence Newsletter/fall2 012ecfe.pdf

[20] S. Almazrooa, S. Woo. Bisphosphonate and nonbisphosphonate-associated osteonecrosis of the jaw: A review. JADA. 2009;140(7):864 -875. Retrieved from http://jada.ada.org/content/140/7/864.full

[21] M. Glick. Closing in on the puzzle of ONJ. JADA. 2008;139(1):12 - 15 . Retrieved from http://jada.ada.org/content/139/1/12.short

[22] M. Drake, B. Clarke, S. Khosla. Bisphosphonates: Mechanism of action and role in clinical practice. Mayo Clin Proc. 2008;83(9):1032-1045. doi:10.4065/83.9.1032
[23] G. Skrepnek, B. Seal, M. Tangirala, M. Jeffcoat, N. Watts, W. Hay. Adverse events and intravenous versus oral bisphosphonate use in patients with osteoporosis and cancer in the U.S. J AGD. 2010;58(6):484-492. Retrieved from http://agd.org/publications/articles/?ArtID=8568

[24] J. Hellstein, R. Adler, B. Edwards, P. Jacobsen, J. Kalmar, S. Koka, J. Migliorati, H. Ristic. Managing the care of patients receiving antiresorptive therapy for prevention and treatment of osteoporosis. JADA. 2011;142(11):1243 -1251. Retrieved from http://jada.ada.org/content/142/11/1243.full

[25] J. Soares, F. Silveira, E. Nunes. Apical surgery with calcium hydroxide capping of the exposed dentine: A case report. J Oral Sci. 2007;49(1):79-83. Retrieved from http://jos.dent.nihon-u.ac.jp/journal/49/1/79.pdf

[26] A. Vernieks, L. Messer. Calcium hydroxide induced healing of periapical lesions: A study of 78 non - vital teeth. Int Endodon J. 1978;11(2):61-69. doi:10.1111/j.1365-2591.1978.tb00663.x

[27] N. Vogt-Ferrier, M. Hugentobler, B. Uebelhart, M. Tramèr, V. Rollason. Interventions for treating osteonecrosis of the jaw bones associated with bisphosphonates. Cochrane Oral Health Group. 2010;6:1-12. doi:10.1002/14651858.CD008455

[28] N. Watts, D. Diab. Long-term use of bisphosphonates in osteoporosis. J Clin Endocrinol Metab. 2010;95(4):1555-1565. doi:10.1210/jc.2009-1947

[29] R. Volker. Osteoporosis screening may be needed less often than previously believed. JAMA. 2012;307(7):654. doi:10.1001/jama.2012.129

[30] M. Gourlay, J. Fine, J. Preisser, R. May, C. Li, L-Y. Lui, D. Ransohoff, J. Cauley, K. Ensrud. Bone-density testing interval and transition to osteoporosis in older women. N Engl J Med. 2012;366(3):225-233. doi:10.1056/NEJMoa1107142

[31] M. Cauley, L-Y. Lui, L. Palermo, K. Ensrud, T. Hiller, M. Nevitt, S. Cummings. Long-term risk of incident vertebral fractures. JAMA. 2007;298(23):2761-2767. doi: 10.1001/jama.298.23.2761

[32] DY. Shin, CR. Ku, KM. Kim, HS. Choi, Y. Rhee, EJ. Lee, S-K. Lim. Spontaneous Non-Traumatic Stress Fractures in Bilateral Femoral Shafts in a Patient Treated with Bisphosphonates. Korean J Intern Med. 2012;27(1):98-102. doi:10.3904/kjim.2012.27.1.98

[33] J. Schneider. Bisphosphonates and low-impact femoral fractures: Current evidence on alendronate-fracture risk. Geriatrics. 2009;64(1):18-23.

[34] C. Menezes-Aguiar, D. de Andrade-Mendes, A. Cruz-Camara. Horizontal root fracture in a maxillary central incisor: a case report. J AGD. 2013;61(2):12-14. 